\title{
Reverse right ventricular remodeling after pulmonary endarterectomy
}

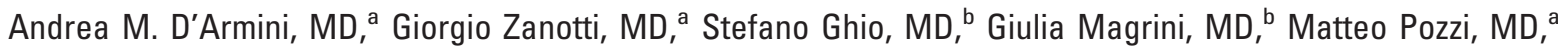 \\ Laura Scelsi, MD, ${ }^{\mathrm{b}}$ Giulia Meloni, MD, ${ }^{\mathrm{c}}$ Catherine Klersy, MD, ${ }^{\mathrm{d}}$ and Mario Viganò, MDa
}

From the Division of Cardiac Surgery, ${ }^{\mathrm{a}}$ the Division of Cardiology, ${ }^{\mathrm{b}}$ the Institute of Radiology, ${ }^{\mathrm{c}}$ and the Biostatistics Unit, ${ }^{\mathrm{d}}$ University of Pavia School of Medicine, Fondazione IRCCS San Matteo Hospital, Pavia, Italy.

Read at the Eighty-sixth Annual Meeting of The American Association for Thoracic Surgery, Philadelphia, Pa, April 29-May 3, 2006.

Received for publication April 24, 2006; revisions received Aug 27, 2006; accepted for publication Aug 31, 2006.

Address for reprints: Andrea M. D'Armini, MD, Division of Cardiac Surgery, University of Pavia School of Medicine, Fondazione IRCCS San Matteo Hospital, Piazzale Golgi 19, 27100 Pavia, Italy (E-mail: darmini@smatteo.pv.it).

J Thorac Cardiovasc Surg 2007;133:162-8

$0022-5223 / \$ 32.00$

Copyright (๑) 2007 by The American Association for Thoracic Surgery

doi:10.1016/j.jtcvs.2006.08.059
Objectives: We sought to evaluate the capability of the right ventricle to regain normal morphology and function after pulmonary endarterectomy, to correlate right ventricular reverse remodeling with functional status, and to identify independent predictors of clinical failure after surgical intervention.

Methods: From December 2000 through August 2003, 45 patients underwent isolated pulmonary endarterectomy. Morphology and function of the right ventricle were studied by using a combination of right heart catheterization, cardiac magnetic resonance, and transthoracic echocardiography. Functional status was evaluated by using New York Heart Association class. Full preoperative data were available for 37 candidates. All patients were evaluated before discharge, at 3 months, and at 1 , 2 , and 3 years postoperatively using the same modalities.

Results: Immediately after surgical intervention, right ventricular cavitary dimensions decreased significantly, and tricuspid regurgitation radically improved. Right ventricular ejection fraction and functional status improved and right ventricular hypertrophy reversed over a longer time period. Higher ventricular dimensions and lower ejection fraction of the right ventricle were associated with poorer functional status at any time postoperatively. At discharge, pulmonary vascular resistance of greater than 509 dyne $\cdot \mathrm{sec} \cdot \mathrm{cm}^{-5}$ and right ventricular ejection fraction of $24 \%$ or less predicted clinical failure at 12 months' follow-up.

Conclusions: After pulmonary endarterectomy, the right ventricle recovers and maintains normal architecture and function over time, regardless of the severity of preoperative disease. Accurate preoperative evaluation of the hemodynamics and anatomy of the thromboembolic lesions are mandatory. If pulmonary endarterectomy is not expected to decrease pulmonary vascular resistance to less than 509 dyne $\cdot \mathrm{sec} \cdot \mathrm{cm}^{-5}$, indication for surgical intervention needs to be carefully evaluated.

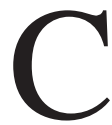
hronic thromboembolic pulmonary hypertension (CTEPH) is a progressive and potentially lethal disease that profoundly affects the morphology and function of the right ventricle. Long-term survival and functional status are dependent on the degree of pulmonary hypertension and pulmonary vascular resistance (PVR) at diagnosis, ${ }^{1,2}$ which are major determinants of right ventricular function. Pulmonary endarterectomy (PEA) is the treatment of choice, is technically feasible in more than $70 \%$ of patients, ${ }^{3}$ and is hemodynamically successful in the vast majority of those undergoing the procedure..$^{3-5}$ Because PEA relieves pulmonary hypertension, we sought to assess the capability of the right ventricle to regain physiologic morphology and function after surgical intervention, which we refer to as reverse remodeling; to assess the durability of these changes; and to correlate right ventricular reverse remodeling with functional status. Also, we tried to identify postoperative predictors of clinical failure. 


\section{Abbreviations and Acronyms \\ $\mathrm{CTEPH}=$ chronic thromboembolic pulmonary hypertension \\ NYHA $=$ New York Heart Association \\ PEA = pulmonary endarterectomy \\ PVR = pulmonary vascular resistance}

\section{Patients and Methods \\ Patients}

From December 2000 through August 2003, 63 patients were evaluated for PEA at our institution. Surgical intervention was indicated in 47 patients (operability rate, $74.6 \%$ ); 45 of these individuals underwent isolated PEA (1 patient refused intervention and 1 died during preoperative evaluation). Preoperative characteristics, operative procedures, hospital outcomes, and follow-up data were prospectively entered into the Pavia Pulmonary Endarterectomy Registry. Use of this registry for research was approved by the hospital review board. Informed consent was obtained for each patient included in the study. The morphology and function of the right ventricle were studied in each patient by using a combination of right heart catheterization, cardiac magnetic resonance, and transthoracic echocardiography. Also, left ventricular data were obtained by means of cardiac magnetic resonance and transthoracic echocardiography to study ventricular interdependence. A full set of preoperative data was available for 37 candidates, who represent our initial study population (Table 1).

\section{Indications and Operative Technique}

The selection criteria for patients undergoing PEA and operative technique have been thoroughly described by Jamieson and kapelanski. ${ }^{5}$ Although the vast majority of our patients had clinically significant tricuspid regurgitation, tricuspid repair was not performed because the incontinence was always deemed functional.

\section{Right Heart Catheterization}

The procedure was performed through the right internal jugular vein by using a flow-directed, balloon-tipped Swan-Ganz catheter (7.5F; Edwards Lifesciences, Irvine, Calif). Measured parameters were cardiac output, as well as right atrial, right ventricular, pulmonary artery, and capillary wedge pressures. Calculated parameters were PVR and cardiac index.

\section{Cardiac Magnetic Resonance}

Examinations were performed with use of a 1.5-T unit (Siemens Magnetom, Malvern, Pa). Hearts were imaged with a body coil and by means of spin echo sequences for morphologic evaluation. Right ventricular measurements were obtained from the 4-chamber transverse plane, and left ventricular measurements were obtained from the short-axis plane. Volumes were measured by using the Argus Function, a fully automated segmentation of high-contrast TrueFISP cine. For each ventricle, the ejection fraction was calculated as follows: [(End-diastolic volume - End-systolic volume)/ End-diastolic volume] $* 100$. Right ventricular free-wall thickness was measured to assess the degree of hypertrophy.
TABLE 1. Patient characteristics

\begin{tabular}{|c|c|c|}
\hline Characteristic & No. & Percentage \\
\hline \multicolumn{3}{|l|}{ Demography } \\
\hline Sex & $25 \mathrm{M} / 12 \mathrm{~F}$ & \\
\hline Age $(y)^{*}$ & $54 \pm 14(21-72)$ & \\
\hline \multicolumn{3}{|l|}{ Risk factors (history) } \\
\hline DVT & 30 & 81.1 \\
\hline $\mathrm{PE}$ & 37 & 100.0 \\
\hline Autoantibodies $†$ & 12 & 32.4 \\
\hline \multicolumn{3}{|l|}{ Functional symptoms } \\
\hline \multicolumn{3}{|l|}{ NYHA } \\
\hline II & 1 & 2.7 \\
\hline III & 13 & 35.1 \\
\hline IV & 23 & 62.2 \\
\hline NYHA III/IV duration (mo)‡ & $17(12-27)$ & \\
\hline NYHA III/IV >1 y & 26 & 70.3 \\
\hline
\end{tabular}

$D V T$, Deep venous thrombosis; $P E$, pulmonary embolism; NYHA, New York Heart Association functional class. $*$ Mean \pm standard deviation (range). $\dagger$ One or more of the following: anticardiolipin antibody, lupus anticoagulant antibody, and antiphospholipid antibody. ¥Median and 25th and 75th percentiles.

\section{Transthoracic Echocardiography}

Standard M-mode, 2-dimensional, and Doppler studies were performed with use of a commercially available echocardiography machine (2.5 MHz probe; Vingmed GE System 5, Horten, Norway). As an indirect index of right ventricular radial systolic function, the right ventricular fractional area shrinkage was calculated as follows: [(Enddiastolic area - End-systolic area)/End-diastolic area ${ }^{*} 100$. The maximum systolic displacement of the lateral portion of the tricuspid annular plane was measured on the M-mode tracing with 2-dimensional echocardiographic guidance to evaluate the right ventricular longitudinal systolic function. Tricuspid regurgitation was graded by using the jet area method. ${ }^{6}$ The tricuspid valve was studied in multiple views to differentiate functional from organic regurgitation. The inferior vena caval diameter was measured from the subcostal view. Its inspiratory collapse was assessed and considered preserved if greater than 50\%. To demonstrate abnormal diastolic leftward interventricular septal motion, we used the end-diastolic eccentricity index. An end-diastolic eccentricity index of greater than 1 was considered pathologic. $^{7}$

Functional Status and Postoperative Clinical Failure

Functional status was evaluated at each time point by using the New York Heart Association (NYHA) classification. At discharge, functional status was not evaluated. Clinical failure was defined as persistence of NYHA class III or IV or death from right ventricular failure 1 year postoperatively.

\section{Follow-up}

All patients were evaluated before discharge and then at 3 months and 1,2 , and 3 years postoperatively by means of right heart catheterization, cardiac magnetic resonance, and transthoracic echocardiography, all performed at our institution over a 2-day period at each time point. Results were assessed in a masked manner. Study follow-up ended in February 2006. Follow-up was complete for 35 patients at discharge and at 3 months, 34 patients at 1 and 2 years, and 21 patients at 3 years. 
TABLE 2. Hemodynamic, cardiac magnetic resonance, echocardiographic variables, and functional symptoms time course

\begin{tabular}{|c|c|c|c|c|c|}
\hline Variable & Preoperative, $\mathbf{n}=37$ & Discharge, $n=35$ & $3 \mathrm{mo}, \mathrm{n}=35$ & $12 \mathrm{mo}, \mathrm{n}=34$ & $24 \mathrm{mo}, \mathrm{n}=34$ \\
\hline PVR (dyne $\left.\cdot \mathrm{sec} \cdot \mathrm{cm}^{-5}\right) \dagger$ & $1143(782-1385)$ & $296(182-409)$ & $238(170-392)$ & 267 (157-429) & $256(168-427)$ \\
\hline $\mathrm{CO}(\mathrm{L} / \mathrm{min})$ & $3.2 \pm 0.8$ & $4.9 \pm 1.3$ & $4.9 \pm 1.4$ & $4.7 \pm 1.0$ & $4.6 \pm 1.1$ \\
\hline $\mathrm{Cl}\left(\mathrm{L} \cdot \min ^{-1} \cdot \mathrm{m}^{-2}\right)$ & $1.7 \pm 0.4$ & $2.7 \pm 0.6$ & $2.6 \pm 0.5$ & $2.5 \pm 0.4$ & $2.4 \pm 0.5$ \\
\hline $\operatorname{RAP}(\mathrm{mm} \mathrm{Hg}) \dagger$ & $7(3-11)$ & $2(0-5)$ & $1(0-3)$ & $2(1-4)$ & $2(1-6)$ \\
\hline RV-ESV (mL) & $80 \pm 33$ & $54 \pm 22$ & $45 \pm 19$ & $42 \pm 13$ & $59 \pm 38$ \\
\hline RV-EF $(\%)$ & $30 \pm 11$ & $33 \pm 9$ & $39 \pm 12$ & $44 \pm 10$ & $46 \pm 11$ \\
\hline RV-WT (mm) & $8.4 \pm 1.3$ & $7.8 \pm 1.3$ & $6.9 \pm 1.2$ & $6.3 \pm 0.8$ & $5.8 \pm 0.8$ \\
\hline LV-EDV (mL) & $88 \pm 29$ & $108 \pm 31$ & $103 \pm 22$ & $107 \pm 19$ & $117 \pm 29$ \\
\hline LV-ESV (mL) & $50 \pm 21$ & $62 \pm 20$ & $56 \pm 16$ & $52 \pm 17$ & $54 \pm 20$ \\
\hline TAPSE (mm) & $15 \pm 4$ & $11 \pm 2$ & $14 \pm 3$ & $15 \pm 2$ & $16 \pm 3$ \\
\hline IVC diameter (mm) & $22 \pm 6$ & $17 \pm 4$ & $15 \pm 4$ & $14 \pm 3$ & $15 \pm 3$ \\
\hline IVC collapsibility & $15(40.5 \%)$ & $31(88.6 \%)$ & $32(91.4 \%)$ & $33(97.1 \%)$ & $30(88.2 \%)$ \\
\hline $\mathrm{EDEI}>1$ & $33(89.2 \%)$ & $5(14.3 \%)$ & $5(14.3 \%)$ & $6(17.6 \%)$ & $7(20.6 \%)$ \\
\hline \multicolumn{6}{|l|}{ NYHA class } \\
\hline III/IV & $36(97.3 \%)$ & NA & $5(14.3 \%)$ & $2(5.9 \%)$ & $4(11.8 \%)$ \\
\hline
\end{tabular}

$P V R$, Pulmonary vascular resistance; $m P A P$, mean pulmonary artery pressure; $C O$, cardiac output; $C l$, cardiac index; $R A P$, right atrial pressure; $C M R$, cardiac magnetic resonance; $R V-E D V$, right ventricular end-diastolic volume; $R V$ - $E S V$, right ventricular end-systolic volume; $R V-E F$, right ventricular ejection fraction; $R V-W T$, right ventricular wall thickness; $L V$ - $E D V$, left ventricular end-diastolic volume; $L V$ - $E S V$, left ventricular end-systolic volume; $L V$ - $E F$, left ventricular ejection fraction; $R V-F A S$, right ventricular fraction of area shrinkage; TR, tricuspid regurgitation; TAPSE, tricuspid annular plane systolic excursion; IVC, inferior vena cava; EDEl, end-diastolic eccentricity index; NYHA, New York Heart Association functional class. *For Bonferroni correction significance if $<.0125$ (4 comparisons) or $<.010$ ( 5 comparisons). $\dagger$ Log transformed to be fitted into the regression model. $\$$ Tricuspid regurgitation $=2$ if the valvular regurgitation area filled one third to two thirds of the right atrium; tricuspid regurgitation $=3$ when a jet filled greater than two thirds of the right atrial area or when systolic reflux in the hepatic veins was detectable. §Preoperative versus 3 months.

\section{Statistical Analysis}

Mean and standard deviation or median and interquartile range (if skewed) were used to describe continuous variables. Counts and percentages were used to describe categoric variables. Changes from baseline and their $95 \%$ confidence intervals were computed. General linear regression models were fitted to assess changes in NYHA class, hemodynamic variables, findings on cardiac magnetic resonance, and echocardiographic characteristics over time. Huber/White/sandwich robust standard errors were computed to account for the dependence between repeated measures in the same patient. The association between NYHA class and right ventricular function and morphology was assessed by using a logistic model for repeated measures with calculation of robust standard errors. A multivariable logistic model was fitted by including all variables with a $P$ value of less than .1 on univariate analysis. The $\mathrm{c}$ statistic (for discrimination) and the shrinkage coefficient (for calibration) were computed for model validation. Finally, receiver operating characteristic curve analysis was performed to identify the optimal cutoff points for postoperative right ventricular ejection fraction and PVR in predicting clinical failure at 12 months.

All analyses were performed with Stata 9.1 software (Stata
Corp, College Station, Tex). The Bonferroni correction for multiple tests was used for post-hoc comparisons.

\section{Results}

\section{Operative Outcomes and Late Deaths}

There was 1 early death after discharge (postoperative day 12) caused by cardiac tamponade, and 1 patient underwent bilateral lung transplantation (postoperative day 4) because of refractory acute right ventricular failure. In the postoperative period, 2 patients had acute right ventricular failure and 3 had reperfusion edema. The median stay in the intensive care unit was 5 days, whereas the median hospital length of stay was 10 days. Over the entire study period, no patients underwent reintervention because of recurrence of CTEPH. One late noncardiac death (secondary to cancer) occurred at 8 months' follow-up.

\section{Hemodynamic Efficacy of PEA}

PEA provided immediate and effective relief from pulmonary hypertension (Table 2): at discharge, PVR was de- 
TABLE 2. Continued

\begin{tabular}{|c|c|c|c|c|c|c|}
\hline \multirow[b]{2}{*}{$36 \mathrm{mo}, \mathrm{n}=21$} & \multirow[b]{2}{*}{$P$ value over time } & \multicolumn{5}{|c|}{$P$ value for post-hoc comparison* } \\
\hline & & $\begin{array}{l}\text { Preoperative vs } \\
\text { discharge }\end{array}$ & Discharge vs 3 mo & $3 \mathrm{mo}$ vs $12 \mathrm{mo}$ & $12 \mathrm{mo}$ vs $24 \mathrm{mo}$ & $24 \mathrm{mo}$ vs $36 \mathrm{mo}$ \\
\hline 306 (192-599) & $<.001$ & $<.001$ & $>.2$ & .164 & $>.2$ & .103 \\
\hline $21(16-35)$ & $<.001$ & $<.001$ & $>.2$ & .084 & $>.2$ & $>.2$ \\
\hline $4.2 \pm 0.8$ & $<.001$ & $<.001$ & $>.2$ & .155 & $>.2$ & .032 \\
\hline $2.2 \pm 0.3$ & $<.001$ & $<.001$ & $>.2$ & .039 & $>.2$ & .022 \\
\hline $2(1-5)$ & $<.001$ & $<.001$ & .009 & .074 & $>.2$ & $>.2$ \\
\hline $96 \pm 43$ & $<.001$ & $<.001$ & .133 & $>.2$ & .002 & $>.2$ \\
\hline $50 \pm 28$ & $<.001$ & $<.001$ & .019 & $>.2$ & .015 & $>.2$ \\
\hline $50 \pm 9$ & $<.001$ & .186 & .001 & .016 & .139 & $>.2$ \\
\hline $5.6 \pm 0.8$ & $<.001$ & .028 & $<.001$ & $<.001$ & .033 & $>.2$ \\
\hline $113 \pm 33$ & $<.001$ & $<.001$ & $>.2$ & $>.2$ & .065 & $>.2$ \\
\hline $52 \pm 25$ & $<.001$ & $<.001$ & .044 & .096 & $>.2$ & $>.2$ \\
\hline $56 \pm 11$ & $<.001$ & $>.2$ & .130 & .008 & $>.2$ & $>.2$ \\
\hline $38 \pm 10$ & $<.001$ & .001 & $>.2$ & .075 & .012 & .032 \\
\hline $7(33.3 \%)$ & $<.001$ & $<.001$ & .149 & $>.2$ & $>.2$ & .031 \\
\hline $17 \pm 3$ & $<.001$ & $<.001$ & $<.001$ & .017 & .064 & $>.2$ \\
\hline $17 \pm 5$ & $<.001$ & $<.001$ & .001 & $>.2$ & .113 & .059 \\
\hline $16(76.2 \%)$ & $<.001$ & $<.001$ & $>.2$ & $>.2$ & $>.2$ & .048 \\
\hline $3(14.3 \%)$ & $<.001$ & $<.001$ & $>.2$ & $>.2$ & $>.2$ & $>.2$ \\
\hline $3(14.3 \%)$ & $<.001$ & & $<.001 \S$ & .200 & .160 & $>.2$ \\
\hline
\end{tabular}

creased by almost $70 \%$, and mean pulmonary artery pressure was decreased by almost $50 \%$ compared with the preoperative values. Cardiac index and cardiac output changed accordingly, whereas central venous pressure normalized. All of these hemodynamic variables remained stable over the entire study period.

\section{Right Ventricular Response to Chronic Pressure Overload Relief}

Immediately after the operation, right ventricular cavitary dimensions were significantly decreased, whereas left ventricular systolic and diastolic volumes were increased (Table 2). In contrast, right ventricular wall thickness remodeled over a longer period of time, and even though decreases in wall thickness were significant only during the first 12 months after the operation, a trend of steady thinning was maintained for the entire study period (Table 2).

Global right ventricular systolic function, as expressed by the right ventricular ejection fraction measured by means of cardiac magnetic resonance, was significantly improved at the third month of follow-up; this variable also continued to show steady progress over the following 3 years, although this did not reach statistical significance (Table 2). The longitudinal systolic function returned to preoperative values within 3 months after a transient decrease immediately after the operation, whereas the radial systolic function was significantly increased at discharge evaluation and continued to improve throughout follow-up (although not statistically significant). Left ventricular ejection fraction moderately increased over the entire study period, reaching statistical significance only at 1 year's follow-up.

Immediately after the operation, tricuspid regurgitation radically improved. Also, the inferior vena cava regained full collapsibility, and its diameter greatly decreased, with no significant change with future measurements (Table 2). In the first few postoperative days, the abnormal interventricular septum displacement rapidly reversed in the majority of patients, and the geometry of the left ventricle steadily improved, with a greater and more protracted increase in end-diastolic volumes compared with end-systolic volumes (Table 2).

\section{Functional Status}

After discharge, functional status steadily improved over the first 12 months of follow-up, with no late changes. Throughout the entire follow-up period, no patients died from cardiac causes.

\section{Postoperative Determinants of Clinical Failure}

By using univariate analysis, higher right ventricular dimensions, lower left ventricular end-diastolic volume, and lower right and left ventricular ejection fraction (measured at each 
TABLE 3. Independent postoperative determinants of clinical failure: Univariable and multivariable analysis

\begin{tabular}{|c|c|c|c|c|c|c|}
\hline \multirow[b]{2}{*}{ Characteristic } & \multirow[b]{2}{*}{$\begin{array}{c}\text { NYHA I-II, } \\
\text { no. visit = } 116\end{array}$} & \multirow[b]{2}{*}{$\begin{array}{c}\text { NYHA III-IV, } \\
\text { no. visit }=57\end{array}$} & \multicolumn{2}{|c|}{ Univariable analysis } & \multicolumn{2}{|c|}{ Multivariable analysis* } \\
\hline & & & $\begin{array}{l}\text { Odds ratio } \\
(95 \% \mathrm{CI}) \dagger\end{array}$ & $P$ valuet‡ & $\begin{array}{l}\text { Odds ratio } \\
(95 \% \mathrm{CI})\end{array}$ & $P$ value \\
\hline \multicolumn{7}{|l|}{ Hemodynamics } \\
\hline PVR (dyne $\left.\cdot \mathrm{sec} \cdot \mathrm{cm}^{-5}\right) \S$ & $241(160-380)$ & $1090(782-1333)$ & $46.2(10.3-206.3)$ & $<.001$ & $28.03(1.10-714)$ & .044 \\
\hline mPAP $(\mathrm{mm} \mathrm{Hg}) \S$ & $19(14-27)$ & $48(34-57)$ & $63.3(10.9-369.3)$ & $<.001$ & & \\
\hline $\mathrm{CO}$ (L/min) & $4.8 \pm 1.1$ & $3.3 \pm 0.8$ & $0.2(0.1-0.3)$ & $<.001$ & $0.47(0.17-1.32)$ & .154 \\
\hline $\mathrm{Cl}\left(\mathrm{L} \cdot \mathrm{min}^{-1} \cdot \mathrm{m}^{-2}\right)$ & $2.5 \pm 0.4$ & $1.8 \pm 0.4$ & $0.02(0.01-0.10)$ & $<.001$ & & \\
\hline RAP $>2$ vs $\leq 2$ & $37(31.9 \%)$ & $37(64.9 \%)$ & $5.8(2.6-13.2)$ & $<.001$ & $0.52(0.10-2.78)$ & .443 \\
\hline \multicolumn{7}{|l|}{ CMR } \\
\hline RV-EDV (mL) & $83 \pm 35$ & $111 \pm 41$ & $1.02(1.01-1.03)$ & $<.001$ & & \\
\hline RV-ESV (mL) & $46 \pm 22$ & $79 \pm 34$ & $1.04(1.02-1.07)$ & $<.001$ & & \\
\hline RV-EF (\%) & $45 \pm 11$ & $30 \pm 11$ & $0.88(0.84-0.93)$ & $<.001$ & & \\
\hline RV-WT (mm) & $6.4 \pm 1.1$ & $8.1 \pm 1.3$ & $3.3(2.0-5.4)$ & $<.001$ & & \\
\hline LV-EDV (mL) & $107 \pm 26$ & $89 \pm 27$ & $0.97(0.95-0.98)$ & $<.001$ & $1.00(0.99-1.01)$ & .842 \\
\hline LV-ESV (mL) & $54 \pm 19$ & $50 \pm 20$ & $0.99(0.97-1.01)$ & .228 & & \\
\hline LV-EF $(\%)$ & $51 \pm 10$ & $44 \pm 13$ & $0.94(0.91-0.98)$ & .01 & & \\
\hline \multicolumn{7}{|l|}{ Echocardiography } \\
\hline RV-EDD (mm) & $31 \pm 5$ & $40 \pm 9$ & $1.2(1.1-1.4)$ & $<.001$ & $0.97(0.87-1.11)$ & .820 \\
\hline RV-FAS (\%) & $38 \pm 11$ & $24 \pm 9$ & $0.9(0.8-0.9)$ & $<.001$ & $0.92(0.86-0.99)$ & .042 \\
\hline TR grade $2-3$ vs $0-1$ & $18(15.5 \%)$ & $39(68.4 \%)$ & $19.3(6.9-54.2)$ & $<.001$ & $1.23(0.21-7.29)$ & .816 \\
\hline TAPSE (mm) & $16 \pm 3$ & $14 \pm 4$ & $0.9(0.7-1)$ & .038 & $1.18(0.93-1.50)$ & .181 \\
\hline IVC diameter (mm) & $15 \pm 3$ & $22 \pm 6$ & $1.5(1.2-1.7)$ & $<.001$ & & \\
\hline IVC collapsibility, yes vs no & $101(87.1 \%)$ & $25(43.9 \%)$ & $0.04(0.01-0.12)$ & $<.001$ & & \\
\hline $\mathrm{EDEI}>1$ & $14(12.1 \%)$ & $40(70.2 \%)$ & $37.39(13.60-106.0)$ & $<.001$ & & \\
\hline
\end{tabular}

$N Y H A$, New York Heart Association functional class; $C l$, confidence interval; $P V R$, pulmonary vascular resistance; $m P A P$, mean pulmonary artery pressure; $C O$, cardiac output; $C l$, cardiac index; $R A P$, right atrial pressure; $C M R$, cardiac magnetic resonance; $R V$ - $E D V$, right ventricular end-diastolic volume; $R V$ - $E S V$, right ventricular end-systolic volume; $R V-E F$, right ventricular ejection fraction; $R V$ - $W T$, right ventricular wall thickness; $L V$ - $E D V$, left ventricular end-diastolic volume; $L V$-ESV, left ventricular end-systolic volume; $L V$ - $E F$, left ventricular ejection fraction; $R V$ - $E D D$, right ventricular end-diastolic diameter; $R V$ - $F A S$, right ventricular fraction of area shrinkage; TR, tricuspid regurgitation; TAPSE, tricuspid annular plane systolic excursion; IVC, inferior vena cava; EDEI, end-diastolic eccentricity index. *Multivariable Logistic model: $P<.001$. Model validation: discrimination, $\mathrm{c}$ statistic $=0.94$; calibration, shrinkage coefficient $=0.72$. $†$ Based on robust standard errors. $\$$ The association is independent on time of visit. $\S$ Log transformed to be fitted in the regression model.

time point during follow-up) were significantly associated with an increased probability for the patient to be within NYHA functional class III or IV rather than I or II (Table 3). Only left ventricular end-systolic volume did not correlate with NYHA class. By using multivariate analysis, right ventricular fractional area shrinkage and PVR were the only predictors of NYHA class after PEA (Table 3).

By means of receiver operating characteristic analysis, we showed that PVR values of greater than 509 dyne. $\mathrm{sec} \cdot \mathrm{cm}^{-5}$ and a right ventricular ejection fraction of $24 \%$ or less at discharge predicted clinical failure at 12 months' follow-up (PVR: specificity, 96.9\%; sensitivity, 60\%; right ventricular ejection fraction: specificity, $74.2 \%$; sensitivity, $75 \%)$.

\section{Discussion}

CTEPH results in chronic right heart pressure overload, which leads to altered right and left ventricular geometry and hemodynamics. Long-term outcome and functional status are determined by the ability of the right ventricle to remodel in the face of an increased hemodynamic load..$^{8,9}$
We chose to evaluate the extent and time course of anatomic and functional derangements induced by CTEPH that are reversible after PEA to determine how durable this reversal is over time and to determine whether these changes in the right ventricle relate to improved functional status of the patients.

To study the right ventricle, we used a comprehensive approach integrating hemodynamic variables, functional data, and volumetric measurements obtained with a nongeometric technique.

Although previous investigators described cardiac changes occurring early after PEA, ${ }^{10,11}$ there is no evidence of global reverse right ventricular remodeling in short-term and midterm periods after PEA.

We and others previously reported success of PEA in the reduction of pulmonary hypertension in patients with CTEPH. ${ }^{3,12-15}$ Regardless of the severity of preoperative disease in our study population, PVR and mean pulmonary artery pressure returned to normal values, cardiac index markedly increased, and right atrial pressures normalized. These variables remained stable throughout the 
entire follow-up period. Right ventricular reverse remodeling was complete and stable over time in the vast majority of our patients. Thus no patient should be denied PEA based on preoperative hemodynamic impairment.

We showed that full recovery of right and left ventricular geometry is achieved soon after PEA, whereas regression of concentric hypertrophy and restoration of full right and left systolic function require a longer period of time. After correction of severe chronic pressure overload, right ventricular reverse remodeling is not limited by pre-existing ventricular size, shape, and function before correction. We observed a modest increase in right ventricular end-diastolic volume at 2 years' follow-up, as well as an insignificant increase in systolic volume. This can be attributed to the presence of outliers (patients with little improvement) within the population with the longest follow-up.

We showed that patients with CTEPH have a high operability rate $(75 \%)$ and that hemodynamic and functional improvement after PEA is remarkable and stable over time. However, there is a small subgroup of patients who do not benefit from PEA. ${ }^{15,16}$ This subgroup is usually affected by either diffuse chronic microvascular thromboembolism or by a combination of different types of pulmonary hypertension with a predominance of microvascular disease. ${ }^{17}$

We found that the degree of postoperative residual PVR was strongly predictive of postoperative functional impairment, and a predischarge value of greater than 509 dyne $\cdot \mathrm{sec} \cdot \mathrm{cm}^{-5}$ was a predictor of clinical failure at 1 year's follow-up, supporting findings from Jamieson and colleagues. ${ }^{14}$ However, very sick and well-selected patients could be significantly benefited in their quality of life and overall survival, even from a moderate reduction in PVR. Thus surgical intervention should not necessarily be contraindicated in those cases in which pulmonary hypertension is out of balance with the angiographic appearance, unless thromboembolic lesions are very distal and represent only a minor component of the pathology of the patients' pulmonary disease. In cases in which PVR at discharge is greater than 509 dyne $\cdot \mathrm{sec} \cdot \mathrm{cm}^{-5}$, adequate medical therapy should be instituted, and lung transplantation should be considered only in patients with progressive and severe functional and hemodynamic impairment.

To differentiate between the patients with a full recovery and those individuals who only partially benefited from surgical intervention, we defined the persistence of NYHA class III or IV or death from right ventricular failure 1 year postoperatively as clinical failure. However, we believe that in patients with extreme CTEPH, the relative reduction in PVR might be important. Even though the postoperative PVR might not be low enough to warrant a full recovery, these patients could still benefit in terms of survival and long-term functional status compared with those who only receive medical treatment. ${ }^{2}$
In conclusion, right ventricular reverse remodeling occurred and was complete in the vast majority of patients who underwent PEA and was independent of preoperative geometry and function. Although reversal of geometric changes occurred early after surgical intervention, regression of hypertrophy and restoration of normal systolic function took place mainly during the first postoperative year, with a tendency to continue up to the third year of follow-up. The major determinant of right ventricular reverse remodeling is the restoration of physiologic pulmonary artery pressure, a condition achieved by the performance of a successful PEA.

We thank T. M. Egan, University of North Carolina at Chapel Hill, for scientific and editorial assistance.

\section{References}

1. Riedel M, Stanek V, Widimsky J, Prerovsky I. Longterm follow-up of patients with pulmonary thromboembolism: late prognosis and evolution of hemodynamic and respiratory data. Chest. 1982;81:151-8.

2. Lewczuk J, Piszko P, Jagas J, Porada A, Wojciak S, Sobkowicz B, et al. Prognostic factors in medically treated patients with chronic pulmonary embolism. Chest. 2001;119:818-23.

3. D'Armini AM, Zanotti G, Viganò M. Pulmonary endarterectomy: the treatment of choice for chronic thromboembolic pulmonary hypertension. Ital Heart J. 2005;6:861-8.

4. Fedullo PF, Auger WR, Kerr KM, Rubin LJ. Chronic thromboembolic pulmonary hypertension. $N$ Engl J Med. 2001;20:1465-72.

5. Jamieson SW, Kapelanski DP. Pulmonary endarterectomy. Curr Probl Surg. 2000;37:165-252.

6. Suzuki Y, Kambara H, Kadota K, Tamaki S, Yamazato A, Nohara R, et al. Detection and evaluation of tricuspid regurgitation using a real-time, two-dimensional, color-coded, Doppler flow imaging system: comparison with contrast two-dimensional echocardiography and right ventriculography. Am J Cardiol. 1986;57:811-5.

7. Ryan T, Petrovic O, Dillon JC, Feigenbaum HF, Conley MJ, Armstrong $\mathrm{W}$. An echocardiographic index for separation of right ventricular volume and pressure overload. J Am Coll Cardiol. 1985;5:918-24.

8. Kelly MC, Nick HS, Rubin L. The right ventricle in pulmonary hypertension. Coron Artery Dis. 2005;16:13-8.

9. Bristow MR, Lawrence SZ, Lowes BD, Abraham WT, Badesch DB, Groves BM, et al. The pressure-overloaded right ventricle in pulmonary hypertension. Chest. 1998;114(suppl):101S-6S.

10. Thistlethwaite PA, Jamieson SW. Tricuspid valvular disease in the patient with chronic pulmonary thromboembolic disease. Curr Opin Cardiol. 2003;18:111-6.

11. Menzel T, Wagner S, Kramm T, Mohr-Kahaly S, Mayer E, Braeuninger S, et al. Pathophysiology of impaired right and left ventricular function in chronic embolic pulmonary hypertension: changes after pulmonary thromboendarterectomy. Chest. 2000;118:897-903.

12. Dartevelle P, Fadel E, Mussot S, Chapelier A, Herve P, de Perrot M, et al. Chronic thromboembolic pulmonary hypertension. Eur Respir J. 2004;23:637-48.

13. D'Armini AM, Cattadori B, Monterosso C, Klersy C, Emmi V, Piovella $\mathrm{F}$, et al. Pulmonary thromboendarterectomy in patients with chronic thromboembolic pulmonary hypertension: hemodynamic characteristics and changes. Eur J Cardiothorac Surg. 2000;18:696-702.

14. Jamieson SW, Kapelanski DP, Sakakibara N, Maneke GR, Thistlethwaite P, Kerr KM, et al. Pulmonary endarterectomy: experience and lessons learned in 1,500 cases. Ann Thorac Surg. 2003;76:1457-62.

15. Mayer E, Dahm M, Hake U, Schmic FX, Pitton M, Kupferwasser I, et al. Mid-term results of pulmonary thromboendarterectomy for chronic thromboembolic pulmonary hypertension. Ann Thorac Surg. 1996;61: 1788-92. 
16. Hartz RS, Byrne JG, Levitsky S, Park J, Rich S. Predictors of mortality in pulmonary thromboendarterectomy. Ann Thorac Surg. 1996;62: 1255-60.

17. Thistlethwaite PA, Mo M, Madani, Deutsch R, Blanchard D, Kapelanski DP, et al. Operative classification of thromboembolic disease determines outcome after pulmonary endarterectomy. $J$ Thorac Cardiovasc Surg. 2002;124:1203-11.

\section{Discussion}

Dr Michael S. Mulligan (Seattle, Wash). The correlation of postoperative patient functional status and right heart recovery is intuitive but has not been documented so clearly before. You are to be commended for your detailed and long-term follow-up. Those of us who care for these patients and perform this operation have observed these findings, but your documentation of these phenomena will be an important contribution to our understanding of this disease and the physiologic effects of unloading the right heart in general.

There would be tremendous value if these data could be used to predict and not simply document a successful outcome. You have attempted to identify predictors of clinical failure. You had full sets of data on patients preoperatively; however, the variables examined were often postoperative assessments. As such, you might answer whether a patient will experience long-term failure but not whether he or she was an appropriate candidate before the operation. I have a few questions.

First, do you have any insights into how your findings might help refine patient selection? For example, did you make any attempt to correlate your echocardiographic/catheter and MRI findings with other established outcome predictors, such as the type of disease by Jamieson classification and how many of the patients with acute or late failure had type 3 or type 4 disease?

Second, so long as you normalize PVR with relief of anatomic obstructions, right heart function will almost invariably recover, but does your experience allow you to identify any patients in whom the right heart will not recover? This issue sometimes arises in considered isolated lung transplantation for patients with longstanding and severe pulmonary hypertension.

Finally, is there a point at which you believe the severity of left ventricular dysfunction precludes safe PEA, and does that estimate vary when considering lung transplantation for such patients wherein PVR decreases even further? Such information might also be useful in guiding the decision between referral for lung versus heart-lung transplantation.

Again, congratulations on your presentation and your excellent results.

Dr D'Armini. Regarding the first question, in our study we had only 1 patient with type 4 lesions and 3 patients with type 3 lesions. Because of the small number of individuals, we were not able to perform any statistical analyses. However, I believe that patients with type 3 lesions, even if surgically more challenging than patients with type 1 or type 2 lesions, might still regain full anatomic and functional recovery of the right ventricle, as long as the operation is performed well.

With regard to the second question, because our primary objectives were to assess the extent and time course of right ventricular reverse remodeling and to analyze the correlation between right ventricular remodeling and functional status, we did not perform this study. However, I think it might be possible to do using the data that we have in our registry.

About the last question, left ventricular dysfunction, manifested mainly as moderate systolic impairment caused by low left ventricular preload, is a consequence of right ventricular remodeling. With the removal of the pulmonary obstructing lesions, CTEPHinduced changes of the right ventricle reverse, left ventricular preload increases, and left systolic function improves. In our experience left ventricular dysfunction has never been a problem during the postoperative period.

Dr Friedrich Wilhelm Mohr (Leipzig, Germany). I had a little concern looking at your tricuspid valves, even in follow-up. You showed nicely that the right ventricular function improved, but in follow-up all the tricuspid valves you showed had a significant regurgitation. Why do you hesitate to do a simple tricuspid annuloplasty?

Dr D'Armini. We did not perform tricuspid annuloplasty because the tricuspid valve regurgitation was always deemed functional. In fact, after the operation, less than $10 \%$ of patients had residual severe regurgitation, and these patients oftentimes also had high pulmonary pressures. In these instances tricuspid regurgitation might have been helpful in preventing acute right ventricular failure in the perioperative period. 